\title{
Prevention of Pelvic Floor Disorders After Vaginal Birth
}

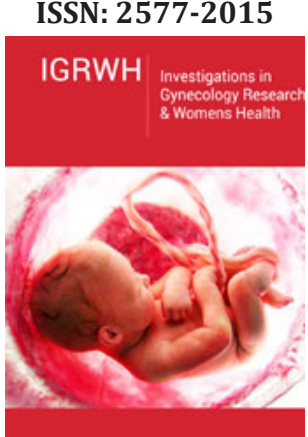

*Corresponding author: Nour Tashtush, Department Pediatrics and Neonatology, Jordan

Submission: 眥January 22, 2020

Published: 想April 15, 2020

Volume 3 - Issue 3

How to cite this article: Nour Tashtush. Prevention of Pelvic Floor Disorders After Vaginal Birth. Invest Gynecol Res Women's Health. 3(3). IGRWH.000565. 2020. DOI: 10.31031/IGRWH.2020.03.000565

Copyright@ Nour Tashtush, This article is distributed under the terms of the Creative Commons Attribution 4.0 International License, which permits unrestricted use and redistribution provided that the original author and source are credited.

\author{
Nour Tashtush* \\ Department Pediatrics and Neonatology, Jordan
}

\begin{abstract}
Vaginal birth is the main risk factor in the development of pelvic floor disorders. Pelvic floor muscle training (PFMT) can prevent prolapse symptoms at 2 years after intervention and could reduce the uptake of treatment. Also, results indicated that PFMT increases the chance of improvement in prolapse stage by $17 \%$ compared to no treatment.
\end{abstract}

Keywords: Vaginal birth; Prolapse; Physical therapy; Pelvic floor muscle strength

Abbreviations: PFMT: Pelvic Floor Muscle Training; TRA: Transverses Abdominis

\section{Introduction}

Epidemiological studies have consistently identified vaginal birth as the main risk factor in the development of pelvic floor disorders. Women who deliver vaginally are 2.8 times more likely to develop stress urinary incontinence and 5.5 times more likely to have pelvic organ prolapse than women who undergo cesarean delivery [1]. The aim of this review article is to assess the importance of pelvic floor training in preventing pelvic floor disorders.

\section{Discussion}

Pelvic organ prolapse is the symptomatic descent of one or more of the anterior vaginal wall, posterior vaginal wall, the uterus (cervix), or the apex of the vagina from the normal anatomical position, caused by herniation through deficient pelvic fascia, or weaknesses or deficiencies in the ligaments or muscles that support the pelvic organs [2]. 5-10 years after a first birth, pelvic floor disorders were dramatically increased among women with a history of at least one operative vaginal birth [3]. Women with normal vaginal delivery and mediolateral episiotomy had the weakest pelvic muscles and nulliparous women had the strongest pelvic muscles [4].

Pelvic floor muscle training can prevent prolapse symptoms at 2 years after intervention and could reduce the uptake of treatment. Therefore, women should be recommended to undertake pelvic floor muscle training even before they have bothersome symptoms. The intervention should be encouraged on the basis of it being safe and done easily by most women [2]. Another conservative method: Pelvic floor physical therapy which targets the muscles, nerves, ligaments, connective tissue, lymphatic system, and joints inside and a rounding the pelvic girdle and focuses on addressing mobility and function. Pelvic floor physical therapists use internal or external therapies such as myofascial release, connective tissue manipulation, joint and scar tissue mobilization. Manual therapy uses palpation to loosen spastic muscles and lengthen tightened tissue to provide relief from pain. Therapies such as neuromuscular electrical stimulation and biofeedback use technology to help women gain functional awareness of the pelvic floor better coordinate muscle contractions and improve endurance to promote maximal functioning [5].

Randomized trials were done to assess the benefit of conservative methods in preventing pelvic prolapse, including six trials. Four trials compared pelvic floor muscle training (PFMT) with no intervention, and two trials compared pelvic floor muscle training plus surgery to surgery alone. PFMT compared to no intervention was found in individual trials to improve prolapse symptoms. Data on prolapsed severity was combined from two trials and results indicated that PFMT increases the chance of improvement in prolapse stage by $17 \%$ compared 
to no treatment. Pelvic floor muscle function appeared to be improved with PFMT in two out of three trials which measured this. Bladder symptoms were improved with PFMT in two out of three trials measuring this; bowel symptoms were measured in one trial, and an improvement with PFMT was found. The two trials which looked at the benefit of PFMT in addition to surgery, were small but of good quality. Findings were contradictory: women benefited from PFMT, in terms of urinary symptoms and pelvic floor muscle strength, in one trial but not the other [6].

Urinary incontinence is associated significantly with pelvic floor disorders, one of the studies on the effect of pelvic floor muscle exercise on quality of life in women with stress urinary incontinence and its relationship with vaginal deliveries has shown Both the combined training of the pelvic floor muscle (PFM) and the synergistic transverses abdominis (TRA) muscle, and the isolated PFM exercises improve the quality of life of women with stress urinary incontinence. Nonetheless, the combined PFM and TRA muscle physiotherapy is more effective. The exercises for the PFM and the synergistic muscle give better results in women who have given birth fewer than three times than isolated PFM exercises [7]. According to the previous mentioned studies, we assure the importance of pelvic floor muscle training as an effective conservative method in preventing and treating pelvic floor disorders.

\section{Conclusion}

Vaginal birth has encountered many cases of pelvic floor disorders that are resulted from the weakening of pelvic floor, so we conclude that preventing measures should be started immediately after the first vaginal birth which include pelvic muscle training and physical therapy that showed positive results as compared to no intervention.

\section{References}

1. Howard D, Makhlouf M (2016) Can pelvic floor dysfunction after vaginal birth be prevented? Int Urogynecol J 27(12): 1811-1815.

2. Hagen S, Glazener C, McClurg D, Macarthur C, Elders A, et al. (2017) Pelvic floor muscle training for secondary prevention of pelvic organ prolapse (PREVPROL): A multicentre randomized controlled trial. Lancet 389(10067): 393-402.

3. Handa L, Blomquist JL, Knoepp LR, Hoskey KA, McDermott KC, et al. (2011) Pelvic floor disorders 5-10 years after vaginal or cesarean childbirth. Obstet Gynecol 118(4): 777-784.

4. Afshari P, Dabagh F, Iravani M, Abedi P (2016) Comparison of pelvic floor muscle strength in nulliparous women and those with normal vaginal delivery and cesarean section. Int Urogynecol J 28(8): 1171-1175.

5. Lawson S, Sacks A (2018) Pelvic floor physical therapy and women's health promotion. J Midwifery Womens Health 63(4): 410-417.

6. Hagen S, Stark D (2011) Conservative prevention and management of pelvic organ prolapse in women. Cochrane Database Syst Rev, Issue 12: CD003882.

7. Magdalena P, Ciećwież S, Brodowska A, Starczewski A, Rutkowska JN, et al. (2018) The effect of pelvic floor muscles exercise on quality of life in women with stress urinary incontinence and its relationship with vaginal deliveries: a randomized trial. BioMed Research International 2019: 1-7. 